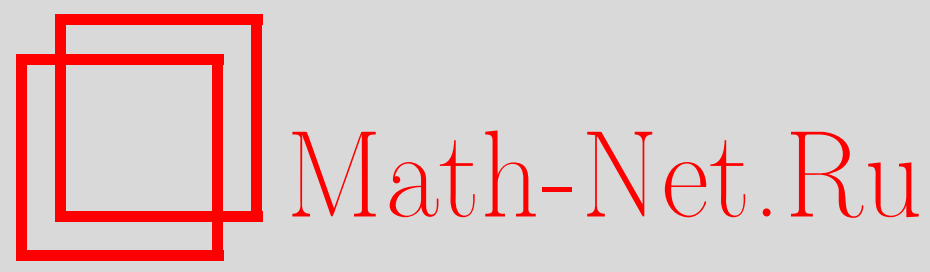

P. Е. Анно, Четырехмерные терминальные горенштейновы факторособенности, Матем. заметки, 2003, том 73, выпуск $6,813-820$

DOI: https://doi.org/10.4213/mzm226

Использование Общероссийского математического портала Math-Net.Ru подразумевает, что вы прочитали и согласны с пользовательским соглашением http://www . mathnet.ru/rus/agreement

Параметры загрузки:

IP: 3.93 .64 .190

26 апреля 2023 г., $15: 28: 17$

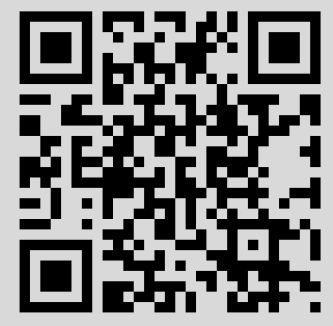




\title{
ЧЕТЫРЕХМЕРНЫЕ ТЕРМИНАЛЬНЫЕ ГОРЕНШТЕЙНОВЫ ФАКТОРОСОБЕННОСТИ
}

\author{
P.Е. Анно
}

\begin{abstract}
В настоящей работе классифицированы все конечные подгруппы $G \subset \mathrm{GL}_{4}(\mathbb{C})$ такие, что фактор $\mathbb{C}^{4}$ по действию $G$ имеет только изолированные терминальные горенштейновы особенности.

Библиограффия: 7 названий.
\end{abstract}

1. Введение. Пусть $(X, p)$ - росток изолированной особенности. Говорят, что особенность терминальна, если $m K_{X}$ является дивизором Картье для некоторого $m \in \mathbb{N}$ и для любого разрешения $f: Y \rightarrow X$ вьполнено

$$
K_{Y} \equiv f^{*} K_{X}+\sum a_{i} E_{i}
$$

где $E_{i}$ - исключительные дивизоры $f$, а все $a_{i}$ больше 0 (см., например, [1]-[3]).

Многообразия с не более чем терминальными особенностями являются минимальным расширением класса гладких многообразий, замкнутьм относительно программы минимальных моделей.

Терминальные факторособенности интенсивно изучались в работах [1], [3]-[5]. Трехмерные терминальные факторособенности полностью классифицированы (см. [1], [3]). В работе [4] классифицированы циклические терминальные горенштейновы факторособенности в размерности 4.

Терминальные факторособенности - достаточно узкий класс особенностей. Однако он довольно удобен для проверки различных гипотез (см. [5]).

В настоящей работе будет доказана (см. пп. 2-5) следующая

Tеорема 1.1. Если конечная подгруппа $G \subset \mathrm{SL}_{4}(\mathbb{C})$ такова, ито фактор $\mathbb{C}^{4}$ по ее действию имеет только терминальные горенштейновы особенности, то имеет место один из следующих случаев:

1) группа $G$ ииклическая, и образующий әлемент имеет собственные значения $\zeta, \zeta^{-1}, \zeta^{a}, \zeta^{-a}$, где а взаимно просто с порядком $G$ (см.м. [4]);

2) группа $G$ изоморфна одной из следующих подгрупп $\mathrm{SU}_{2}(\mathbb{C}): \widetilde{D}_{n}, \widetilde{A}_{4}, \widetilde{S}_{4}, \widetilde{A}_{5}$, а представление есть сумма двух изоморфных неприводимых двумерных представлений;

Работа выполнена при частичной поддержке Российского фонда фундаментальных исследований, грант 02-01-00441, и гранта INTAS-OPEN-2000-269. 
3) группа $G$ изоморфна $\left(\mu_{n} \times G^{\prime}\right) /\{(1,1) ;(-1,-1)\}$, где $G^{\prime}$ - одна из групп, перечисленных в предыдущих пунктах, $n$ взаимно просто с порядком $G^{\prime}$, представление есть сумма двух сопряженных неприводимых двумерных представлений;

4) группа $G$ изоморфна расслоенному произведению $\widetilde{D}_{3 p}, p \in \mathbb{N}$, и $\widetilde{S}_{4}\left(\right.$ или $\left.\widetilde{A}_{4}\right)$ над $D_{3}$ (см. пример 4.1).

СлЕдСтвиЕ 1.2 ([1, раздел 6.4]). Пусть $X:=\mathbb{C}^{4} / G$ - четырехмерная терминальная горенштейнова факторособенность. Тогда общий дивизор $F \in\left|-2 K_{X}\right|$ имеет лишь горенштейновы канонические особенности.

СледСТВИЕ 1.3. Пусть $X:=\mathbb{C}^{4} / G$ - четырехмерная терминальная горенштейнова факторособенность. Тогда особенность $X$ неисключительна [6].

2. Предварительные сведения. Начнем доказательство теоремы $1.1 \mathrm{c}$ некоторых вспомогательных утверждений.

Tеорема 2.1 [4]. Пусть $G \subset \mathrm{GL}_{4}(\mathbb{C})$ - конечная подгруппа, не содер жащая отражсний. Тогда следующие условия әквивалентны:

1) факторособенность $X:=\mathbb{C}^{4} / G$ терминальна и горенштейнова;

2) каждый элемент $g \in G$ имеет собственные значения вида $\left(\varepsilon, \varepsilon^{-1}, \varepsilon^{a}, \varepsilon^{-a}\right)$ для некоторых $\varepsilon \in \mathbb{C}, \varepsilon^{n}=1, \operatorname{gcd}(a, n)=1$.

В случае 2) будем для краткости говорить, что группа $G \subset \mathrm{SL}_{4}(\mathbb{C})$ удовлетворяет условию $(*)$.

Всюду предполагается, что $G \subset \mathrm{GL}_{4}(\mathbb{C})$ - конечная подгруппа, не содержащая отражений.

Тогда задача заключается в том, чтобы классифицировать все конечные подгруппы $\mathrm{SL}_{4}(\mathbb{C})$, удовлетворяющие условию $(*)$.

ПримеР 2.2. Прямая сумма двух изоморфных представлений групшы Клейна в $\mathrm{SL}_{2}(\mathbb{C})$ удовлетворяет условию $(*)$, так как любой элемент имеет собственные значения $\left(\varepsilon, \varepsilon^{-1}, \varepsilon, \varepsilon^{-1}\right)$.

Пусть есть группа $G$, удовлетворяющая условию $(*)$. Предположим, что еепредставление в $\mathrm{SL}_{4}(\mathbb{C})$ приводимо.

ЛЕмма 2.3. Если представление әруппы $G$, удовлетворяющей условию (*), приводимо, то либо G чиклическая, либо это представление есть прямая сумма двух двумерных представлений.

ДокАЗАТЕльство. Если есть инвариантное одномерное подпредставление, то оно задает гомоморфизм $G$ в циклическую подгрупу $\mathbb{C}^{*}$. Ядро этого гомоморфизма тривиально, так как в силу условия $(*)$ неединичный элемент не может иметь собственное значение 1. Лемма доказана.

Разберем подробнее случай прямой суммы двух двумерных представлений.

ПРЕДЛОЖЕНИЕ 2.4. Если прямая сумма $\rho \oplus \rho^{\prime}$ двух двумерных представлений әруппы $G$ удовлетворяет условию (*), то возмохсны два варианта:

1) $G \simeq\left(\mu_{3 p} \times \mu_{3} \widetilde{A}_{4}\right) /\{(1,1) ;(-1,-1)\}($ подробнее см. пример 4.1$)$;

2) $G \simeq\left(\mu_{n} \times G^{\prime}\right) /\{(1,1) ;(-1,-1)\}$, әде $G^{\prime}$ - әруппа Клейна и п взаимно просто с порядком $G^{\prime}$. 
ДокАЗАТЕЛЬСТВо. По условию $(*)$ оба представления $\rho, \rho^{\prime}$ точны, кроме того, для любого $g \in G$ из $(*)$ имеем $\operatorname{tr}\left(\rho \oplus \rho^{\prime}\right)(g) \in \mathbb{R}$, поэтому либо $\operatorname{tr} \rho(g)=\overline{\operatorname{tr} \rho^{\prime}(g)}$ и $\rho^{\prime} \simeq \rho^{\vee}$, либо $\operatorname{tr} \rho(g), \operatorname{tr} \rho^{\prime}(g) \in \mathbb{R}$. Во втором случае сразу получаем второй пункт предложения, так как $\rho: G \rightarrow \mathrm{SL}_{2}(\mathbb{C})$ и можно выбрать $G^{\prime} \simeq G, n=2$. В первом случае исследуем, каким может быть представление $G \simeq \rho(G) \subset \mathrm{GL}_{2}(\mathbb{C})$.

Имеем $\mathrm{GL}_{2}(\mathbb{C})=\left(\mathrm{SL}_{2}(\mathbb{C}) \times \mathbb{C}^{*}\right) /\{(1,1) ;(-1,-1)\}$. Аналогично доказательству утверждения 4.2 ниже получаем, что либо для проекции прообраза $\rho(G)$ в $\mathrm{SL}_{2}(\mathbb{C}) \times \mathbb{C}^{*}$ на первый сомножитель имеет место один из следующих трех случаев:

1) $\mu_{n}$; тогда $G$ - циклическая;

2) $\widetilde{A}_{4}, G \cap \mathrm{SL}_{2} \simeq Q_{8}$; тогда $G$ - подгруппа групшы из примера 4.1 ,

$G=\left(\mu_{3 p} \times \mu_{3} \widetilde{A}_{4}\right) /\{(1,1) ;(-1,-1)\}$

3) $\widetilde{S}_{4}, G \cap \mathrm{SL}_{2} \simeq \widetilde{A}_{4}$; тогда $G \simeq \widetilde{A}_{4} \times \mu_{n}$,

либо прообраз $\rho(G)$ в $\mathrm{SL}_{2}(\mathbb{C}) \times \mathbb{C}^{*}-$ прямое произведение циклической группы и одной из групп Клейна.

В этом случае представление $G$ в $\mathrm{GL}_{2}(\mathbb{C})$ можно продолжить до представления $G$ в $\mathrm{SL}_{4}(\mathbb{C})$, удовлетворяющего условию $(*)$, если и только если

$$
G=\left(\mu_{n} \times G^{\prime}\right) /\{(1,1) ;(-1,-1)\}
$$

и $n$ взаимно просто с порядком групшы Клейна $G^{\prime}$. В этом случае ни один не единичный элемент $G$ не имеет в $\mathrm{GL}_{2}(\mathbb{C})$ собственного значения равного 1 и прямая сумма представления в $\mathrm{GL}_{2}(\mathbb{C})$ и сопряженного к нему является представлением $G$ в $\mathrm{SL}_{4}(\mathbb{C})$, удовлетворяющим условию $(*)$. Предложение доказано.

Пусть теперь представление $G$ неприводимо.

ПРЕДЛОЖЕНИЕ 2.5. Если представление $G$ неприводимо, то $G$ сохраняет невырохденную билинейную форму, либо симметричную, либо кососимметричную.

ДокАЗАтЕльство. Рассматриваемое представление $G$ имеет вещественный характер, значит, изоморфно своему сопряженному. Этот изоморфизм задает невырожденную билинейную форму $(\cdot, \cdot)$ на $\mathbb{C}^{4}$, а так как он коммутирует с действием $G$, эта форма $G$-инвариантна.

Рассмотрим симметричную и кососимметричную части формы $(\cdot, \cdot)$. Каждая из них либо невырождена, либо тождественно равна 0 , так как иначе ее ядро - инвариантное подпредставление. Предложение доказано.

ПРЕДЛОЖЕНИЕ 2.6 [7, п. 4.2.15]. Если конечная группа $G$ лежит в $\mathrm{SO}_{n}(\mathbb{C})$, то $G \subset \mathrm{SO}_{n}(\mathbb{R}) \subset \mathrm{SO}_{n}(\mathbb{C})$ для некоторого вложсения $\mathrm{SO}_{n}(\mathbb{R}) \subset \mathrm{SO}_{n}(\mathbb{C})$.

СлЕдСТВИЕ 2.7. Если конечная группа $G \subset \mathrm{SL}_{4}(\mathbb{C})$ удовлетворяет условию $(*)$, mо либо $G \subset \mathrm{SO}_{4}(\mathbb{R})$, либо $G \subset \mathrm{Sp}_{4}(\mathbb{C})$.

Первьй случай разбирается в п. 4, второй - в п. 5.

3. Группы Клейна. Разберем отдельно случай, когда $G$ - группа Клейна, т.е. одна из следуюших групш: $\mu_{n}, \widetilde{D}_{n}, \widetilde{A}_{4}, \widetilde{S}_{4}, \widetilde{A}_{5}$.

ПРЕДЛОЖЕНИЕ 3.1. У групп Клейна нет 4-мерных неприводимых представлений, удовлетворяющих условию $(*)$. 
ДокАЗАТЕЛЬСТВО. Все неприводимые представления $\mu_{n}$ одномерны.

Группа $\widetilde{D}_{n}$ порождается двумя элементами $r, s$ с соотношениями $r^{n}=s^{4}=1$, $s r s^{-1}=r^{-1}$. В любом представлении $\widetilde{D}_{n}$ образ $s$ переставляет собственные подпространства $r$ с собственными значениями $\varepsilon, \varepsilon^{-1}$ (пусть $r v=\varepsilon v$, тогда $r(s v)=s r^{-1} v=$ $\left.s \varepsilon^{-1} v=\varepsilon^{-1}(s v)\right)$. Элемент $s^{2}$ имеет порядок 2, поэтому в 4-мерном представлении, удовлетворяющем условию (*), он переходит в -1 ; значит, для любого вектора $v$, собственного для $r$, подпространство, натянутое на $v$ и $s v$, будет инвариантно относительно действия $r$ и $s$, а следовательно, и всей группы $\widetilde{D}_{n}$.

Таким образом, у $\widetilde{D}_{n}$ также нет 4 -мерных неприводимых представлений.

Их нет и у $\widetilde{A}_{4}$, это видно из подсчета размерностей неприводимых представлений: сумма квадратов этих размерностей должна быть равна порядку группы. Порядок $\widetilde{A}_{4}$ равен 24 , но только квадраты представлений $A_{4}$ уже дают 12 , значит, 4 -мерного представления нет.

Отсутствие неприводимого 4-мерного представления у $\widetilde{S}_{4}$ и $\widetilde{A}_{5}$ можно установить, вычисляя норму характера. Условие $(*)$ однозначно задает значения характера для элементов порядка $2,3,4$; вычисление показьвает, что этих значений достаточно, чтобы норма оказалась больше единицы.

Предложение доказано.

4. Ортогональный случай. Пусть теперь $G \subset \mathrm{SO}_{4}(\mathbb{R})$.

Заметим, что условие $(*)$ влечет следуюшие свойства:

1. Любая абелева подгруппа $G$ циклическая.

2. Центр $G$ либо тривиален, либо состоит из двух элементов $\{1,-1\}$, так как представление неприводимо, a -1 - единственный скаляр, согласно условию (*).

Таким образом, мы приходим к задаче классификации всех конечных подгруш $\mathrm{SO}_{4}(\mathbb{R})$, удовлетворяющих свойствам 1 и 2 .

ПримеР 4.1. Рассмотрим группу $\Gamma$ - расслоенное произведение $\widetilde{D}_{3 p}, p \in \mathbb{N}$, и $\widetilde{S}_{4}$ над $D_{3}$, которая является фактором $\widetilde{D}_{3 p}$ по $\mu_{2 p}$ и $\widetilde{S}_{4}$ по $Q_{8}-$ коммутанту $\widetilde{A}_{4} \subset \widetilde{S}_{4}$. Оно лежит в $\widetilde{D}_{3 p} \times \widetilde{S}_{4}$, а эта группа имеет представление в $\mathrm{SL}_{4}(\mathbb{C})$, строящееся следующим образом: $\widetilde{D}_{3 p}$ представляется матрицами

$$
\left(\begin{array}{cc}
\varepsilon \cdot \mathrm{Id} & 0 \\
0 & \varepsilon^{-1} \cdot \mathrm{Id}
\end{array}\right) \text { и }\left(\begin{array}{cc}
0 & \varepsilon \cdot \mathrm{Id} \\
\varepsilon^{-1} \cdot \mathrm{Id} & 0
\end{array}\right),
$$

а $\widetilde{S}_{4}-$ матрицами

$$
\left(\begin{array}{cc}
A & 0 \\
0 & A
\end{array}\right)
$$

как прямая сумма двух представлений $\widetilde{S}_{4}$ в $\mathrm{SL}_{2}(\mathbb{C})$. Значит, Г тоже имеет представление в $\mathrm{SL}_{4}(\mathbb{C})$. Легко убедиться, что оно неприводимо.

УТВЕРЖДЕНИЕ 4.2. Если $G \subset \mathrm{SO}_{4}(\mathbb{R})$ и удовлетворяет условию $(*)$, то либо $G$ - одна из групп Клейна, и представление - прямая сумма двух изоморфных неприводимых двумерных, либо $G$ - группа, описанная в примере 4.1.

ДокАЗАТЕЛЬСтво. Рассмотрим $H-$ прообраз $G$ при накрытии $\mathrm{SU}_{2} \times \mathrm{SU}_{2} \rightarrow$ $\mathrm{SO}_{4}(\mathbb{R})$.

Все конечные подгруппы $\mathrm{SU}_{2}$ известны- это группы Клейна $\mu_{n}, \widetilde{D}_{n}, \widetilde{A}_{4}, \widetilde{S}_{4}, \widetilde{A}_{5}$. Обозначим $H_{1}$ и $H_{2}$ проекции $H$ на сомножители в $\mathrm{SU}_{2} \times \mathrm{SU}_{2}$; положим $B_{i}=H \cap H_{i}$. Тогда 
$H \subset H_{1} \times H_{2}$; очевидно, $H_{2}$ является ядром проекции $H_{1} \times H_{2}$ на $H_{1}$, поэтому $B_{2}$ является ядром проекции $H$ на $H_{1}$. Аналогично $B_{1}$ - ядро проекции $H$ на $H_{2}$. Группы $B_{i}$ нормальны в $H$, следовательно, нормальны в $H_{i}$, так как при сюръективном гомоморфизме нормальные подгруппы переходят в нормальные.

Заметим, что если одна из $B_{i}$ тривиальна или равна $\mu_{2}$, то $G=H /\{(1,1),(-1,-1)\}$ изоморфна одной из групп Клейна; если $H_{i}=\mu_{n}$, то $B_{i}$ лежит в центре $H$, и, следовательно, состоит не более, чем из двух элементов.

Перебирая все (немногочисленные) нормальные подгрупшы групп Клейна видим, что единственньй вариант, кроме указанных выше, при котором в $B_{1} \times B_{2} \subset H$ ненарушается свойство 1 (что происходит, когда $B_{1}$ и $B_{2}$ содержат элементы одинакового порядка, отличные от $\{-1,-1\})$, есть вариант $H_{i}=D_{n}, B_{i}=\mu_{p}$.

Заметим, что $H_{1} / B_{1} \simeq H_{2} / B_{2} \simeq H / B_{1} \times B_{2}$, поэтому остаются две возможности:

1) $H_{1}=\widetilde{D}_{p k}, H_{2}=\widetilde{D}_{q k}, B_{1}=\mu_{2 p}, B_{2}=\mu_{2 q},(p, q)=1$, тогда $H=\widetilde{D}_{p q k}$;

2) $H_{1}=\widetilde{D}_{3 p}, H_{2}=\widetilde{S}_{4}, B_{1}=\mu_{2 p}, B_{2}=Q_{8}$; в этом случае $G$ - группа из примеpa 4.1 .

Утверждение доказано.

5. Симплектический случай. Пусть теперь $G \subset \mathrm{Sp}_{4}(\mathbb{C})$.

УТВЕРЖДЕНИЕ 5.1. В этом случае $G$ - группа Клейна и представление - сумма двух изоморфных представлений $G$ в $\mathrm{SL}_{2}(\mathbb{C})$.

Имеется накрытие $\mathrm{Sp}_{4}(\mathbb{C}) \rightarrow \mathrm{SO}_{5}(\mathbb{C})$ с ядром $\{1,-1\}$.

При этом по предложению 2.6 группа $G$ отображается в $\mathrm{SO}_{5}(\mathbb{R}) \subset \mathrm{SO}_{5}(\mathbb{C})$.

Сначала докажем несколько вспомогательных лемм.

ЛЕмма 5.2. Если образ конечной подгруппь $\mathrm{Sp}_{4}$, удовлетворяющей условию (*), при отображении в $\mathrm{SO}_{5}$ имеет инвариантный вектор, то эта подгруппа изоморфна какой-то әруппе Клейна, причем ее представление в $\mathrm{Sp}_{4}$ изоморфно прямой сумме двух одинаковых представлений в $\mathrm{SL}_{2}$.

ДокАЗАтЕЛЬСтво. Если $H \subset \mathrm{Sp}_{4}$ при накрытии $\mathrm{Sp}_{4} \rightarrow \mathrm{SO}_{5}$ отображается в $H^{\prime} \subset \mathrm{SO}_{4}$, это означает, что $H$ сохраняет некий ненулевой вектор в $\mathrm{七}^{2} \mathbb{C}^{4}$, помимо соответствующего основной симплектической форме. Тогда представление $H$ в $\mathbb{C}^{4}$ не может быть неприводимьм, так как оно сохраняет две линейно независимых симплектических формы, которьм соответствуют два линейно независимых изоморфизма $\mathbb{C}^{4}$ и $\left(\mathbb{C}^{4}\right)^{\vee}$, а по лемме Шура любые два изоморфизма неприводимых представлений пропорциональны.

По лемме 2.3 либо группа $H$ циклическая, и тогда все доказано, либо ее представление является суммой двух двумерных представлений, обозначим их $V_{1}$ и $V_{2}$. Ограничение $H$ на любое из этих пространств задает гомоморфизм $H$ в $\mathrm{GL}_{2}$, который из-за условия $(*)$ является вложением.

По доказанному вьше $H$ сохраняет две симплектические формы, обозначим их $(\cdot, \cdot)$ и $(\cdot, \cdot)^{\prime}$. Если хотя бы одна из них невырождена на одном из $V_{1}, V_{2}$, то ограничение действия $H$ на это подпространство задает вложение $H$ в $\mathrm{SL}_{2}(\mathbb{C})$. Если ограничения обеих форм на оба подпространства вырождены, выберем базисы в $V_{1}$ и $V_{2}$ так, чтобы $(\cdot, \cdot)$ приобрела канонический вид

$$
\left(\begin{array}{c|c}
0 & \mathrm{Id} \\
\hline-\mathrm{Id} & 0
\end{array}\right),
$$


где Id - единичная матрица.

Тогда элементы $H$ запишутся в виде

$$
\left(\begin{array}{c|c}
A & 0 \\
\hline 0 & \left(A^{T}\right)^{-1}
\end{array}\right),
$$

а форма $(\cdot, \cdot)^{\prime}-$ в виде

$$
\left(\begin{array}{c|c}
0 & F \\
\hline-F & 0
\end{array}\right),
$$

Группа $H$ сохраняет $(\cdot, \cdot)^{\prime}$; записав это условие явно, получаем, что $F$ как матрица коммутирует с $A$, т.е. образы всех элементов при вложении $H$ в $\mathrm{GL}_{2}$ коммутируют с некоторой матрицей $F$. У $F$ есть собственный вектор; тогда либо он является собственньм и для всех элементов $H$, и тогда по лемме 2.3 групша $H$ циклическая, либо $F-$ скалярная матрища, и тогда формы $(\cdot, \cdot)$ и $(\cdot, \cdot)^{\prime}$ линейно зависимы.

Получается, что $H$ в любом случае вкладьвается в $\mathrm{SL}_{2}(\mathbb{C})$, и утверждение леммы следует теперь из предложения 3.1. Лемма доказана.

ЛЕмма 5.3. Все әлементы $G$ с собственными значениями вида $\left(\varepsilon, \varepsilon^{-1}, \varepsilon, \varepsilon^{-1}\right)$ составляют подгруппу $H$, изоморфную одной из групп Клейна.

ДокАЗАТЕЛьСТво. Эти элементы при описанном отображении $G$ в $\mathrm{SO}_{5}(\mathbb{R})$ переходят в операторы с комплексными собственными значениями $\left(1,1,1, \varepsilon^{2}, \varepsilon^{-2}\right)$, т.е. вращения в некоторой двумерной плоскости. По предыдущей лемме любые два таких оператора порождают некоторую группу Клейна, проецирующуюся в подгруппу $\mathrm{SO}_{3}(\mathbb{R})$, т.е. сохраняющую двумерное подпространство $\mathbb{R}^{5}$. Значит, для любых двух таких операторов упомянутые двумерные плоскости имеют ненулевое пересечение. Значит, все эти плоскости лежат в одном трехмерном подпространстве: выберем из них две разные (они порождают трехмерное подпространство), тогда любая из остальных двумерных плоскостей натянута на свои пересечения с этими двумя выбранными, т.е. лежит в том же 3-мерном подпространстве. Лемма доказана.

ЗАмечАниЕ 5.4. Заметим, что либо групша $H$ циклическая, либо образ $H$ в $\mathrm{SO}_{5}(\mathbb{R})$ сохраняет некоторое двумерное подпространство. Более того, в этом случае размерность инвариантного относительно элемента $g$ подпространства в $\mathbb{R}^{5}$ является критерием принадлежности $g$ к $H$ : если размерность равна 3 , то $g \in H$, если она равна 1 , то нет.

ЛЕмма 5.5. Если $G$, удовлетворяющая условию (*), при отображсении $\mathrm{Sp}_{4} \rightarrow$ $\mathrm{SO}_{5}(\mathbb{R})$ переходит в подгруппу $\mathrm{SO}_{3}(\mathbb{R}) \times \mathrm{SO}_{2}(\mathbb{R})$, то $G$ - группа Клейна.

ДоказАТЕльство. Прообраз $\mathrm{SO}_{3}(\mathbb{R})$ - групша $\mathrm{SU}_{2}(\mathbb{C})$, вложенная в $\mathrm{Sp}_{4}(\mathbb{C})$ в виде прямой суммы двух одинаковых представлений $\mathrm{SU}_{2}(\mathbb{C})$. Можно явно посчитать, что при этом $\mathrm{SO}_{2}(\mathbb{R}) \subset \mathrm{SU}_{2}(\mathbb{C})$ переходит в матрицы, коммутируюшие с прообразом $\mathrm{SO}_{3}(\mathbb{R})$. Рассматривая аналогично доказательству утверждения 4.2 прямое произведение какой-нибудь циклической подгруппы пересечения образа $G$ в $\mathrm{SO}_{5}$ и $\mathrm{SO}_{3}$ на пересечение образа $G$ и $\mathrm{SO}_{2}$, получаем, что это коммутативная подгруппа; ее прообраз в $\mathrm{Sp}_{4}(\mathbb{C})$ коммутативен по написанному вьше, но не цикличен, если проекция на $\mathrm{SO}_{3}$ имеет ядро, т.е. если $G$ не является группой Клейна; противоречие. Лемма доказана.

Лемма 5.6. В случае нециклической $H$ если для әлемента $g$ выполнено $g^{k} \in H$, mо либо $g \in H$, либо $g^{k}= \pm 1$. 
ДокАЗАТЕЛЬСТво. Пусть $H \subset \mathrm{SO}\left(V_{0}\right), \operatorname{dim} V_{0}=3$, действие $g$ раскладьвается в прямую сумму $V_{1} \oplus V_{2} \oplus\langle v\rangle$. Тогда $g^{k}$ стабилизирует $v$ и одно из $V_{1}, V_{2}$. Пусть для определенности $V_{1} \subset V_{0}$, действие $g^{k}$ сохраняет $V_{2} \oplus\langle v\rangle$. Тогда $V_{2} \cap V_{0}^{\perp} \neq \varnothing$; если $V_{2} \neq V_{0}^{\perp}$, обозначим $V_{2} \cap V_{0}^{\perp}=\langle w\rangle$, тогда для любого $h \in H$ элемент $g^{-1} h g$ сохраняет $g^{-1} w$, значит, $g^{-1} H g \subset H$. Отсюда $g^{-1} H g=H$, поэтому $H$ сохраняет $g^{-1} w$, тогда группа $H \subset \mathrm{SO}_{2}$ циклическая. Если же $V_{2}=V_{0}^{\perp}$, то групп, порожденная $H$ и $g$, лежит в $\mathrm{SO}_{3} \times \mathrm{SO}_{2}$ и по предыдущей лемме является группой Клейна, значит, она либо циклическая (но она содержит нециклическую $H$ ), либо вкладывается в $\mathrm{SO}_{3}$, но тогда по замечанию все ее элементы принадлежат $H$. Лемма доказана.

Лемма 5.7. Если әруппа $H$ из леммы 5.3 не ииклическая, то имеет место разлохение

$$
G=\left(\bigcup_{i=1}^{k} H_{i}\right) \cup H
$$

әде $H_{i}$ ииклические, и любъе две группь из $H_{i}, H$ либо совпадают, либо пересекаются только по множеству $\{1,-1\}$; если же $Н$ ииклическал, то имеет место разложение

$$
G=\left(\bigcup_{i=1}^{k} H_{i}\right)
$$

əде $H_{i}$ ииклические, $H_{i} \cap H_{j}=\{1,-1\}$.

ДокАЗАТЕЛьСтво. Выделим $H \subset G$ по лемме 5.3. Для каждого $x \notin H$ возьмем максимальную циклическую подгруппу, содержащую $x$. Если $H$ не циклическая, то эти подгрупшы, во-первых, пересекаются с $H$ только по $\{1,-1\}$ по лемме 5.6, а во-вторых, для разных $x$ либо совпадают, либо пересекаются также по $\{1,-1\}$. (Заметим, что $\{1,-1\}$ действительно принадлежит всем построенным $H_{i}$, так как иначе група, порожденная какой-то из $H_{i}$ и -1 , была бы абелевой, но не циклической.) Каждая циклическая подгрупа $H_{i}$ характеризуется тем, что сохраняет определенньй вектор в $\mathbb{R}^{5}$, единственньй, иначе образующая $H_{i}$ лежит в $H$. Пересечение двух таких подгруп сохраняет вектора их обеих (они разные, так как иначе обе подгруппы лежат в одном экземпляре $\mathrm{SO}_{4}$ и либо лежат в $H$, либо совпадают, так как по построению они максимальные), значит, сохраняет некоторое 3-мерное подпространство, следовательно, лежит в $H$. Но мы уже доказали, что $H_{i}$ и $H$ пересекаются только по $\{1,-1\}$.

Если $H$ циклическая, то она принадлежит только одной из $H_{i}$, так как иначе группа, порожденная $H_{i}, H_{j}$ такими, что $H_{i} \cap H_{j}=H$, принадлежит $\mathrm{SO}_{3} \times \mathrm{SO}_{2}$ и по лемме 5.5 является группой Клейна, т.е. либо циклической, что противоречит построению $H_{i}, H_{j}$, либо подгруппой $\mathrm{SO}_{3}$ и, следовательно, совпадает с $H$.

Лемма доказана.

Теперь докажем собственно предложение 5.1.

ДОКАЗАТЕЛЬСТВО ПРЕДЛОЖЕНИЯ 5.1. Вычислим скалярный квадрат характера нашего 4-мерного представления, пользуясь разложением из леммы 4.7. Если представ- 
ление неприводимо, он должен быть равен 1. Итак, имеем

$$
\begin{aligned}
\frac{1}{\# G} \sum_{g \in G} \chi(g) \overline{\chi(g)}= & \frac{1}{\# G}\left(\sum_{i=1}^{k} \sum_{g \in H_{i}} \chi(g) \overline{\chi(g)}\right. \\
& \left.+\sum_{g \in H} \chi(g) \overline{\chi(g)}-k(\chi(1) \overline{\chi(1)}+\chi(-1) \overline{\chi(-1)})\right) .
\end{aligned}
$$

Вычисляем $\sum_{g \in H_{i}} \chi(g) \overline{\chi(g)}=4 \# H_{i}$, так как в ограничении на $H_{i}$ наше представление дает 4 разных одномерных представления; далее, $\sum_{g \in H} \chi(g) \overline{\chi(g)}=8 \# H$, если $H$ циклическая, так как в ограничении на $H$ наше представление дает 2 пары одинаковых одномерных, и $\sum_{g \in H} \chi(g) \overline{\chi(g)}=4 \# H$, если $H$ не циклическая, так как тогда получается 2 одинаковых неприводимых двумерных.

Заметим, что \#G $=\sum_{i=1}^{k} \# H_{i}+\# H-2 k$. Порядок $H_{i}$ не меньше 10 , так как он должен быть четным, поскольку $H_{i}$ содержит -1 , а любой элемент порядка 4 по условию $(*)$ лежит в $H$, поэтому элементы порядка 8 , если они есть, тоже лежат в $H$.

Скалярный квадрат характера неприводимого представления равен 1 , отсюда получаем, что

$$
\frac{1}{\# G}\left(4 \sum_{i=1}^{k} \# H_{i}+C \cdot \# H-32 k\right)=1
$$

где $C=4$ или 8 ; поэтому

$$
4 \sum_{i=1}^{k} \# H_{i}+C \cdot \# H-32 k=\# G=\sum_{i=1}^{k} \# H_{i}+\# H-2 k .
$$

Вычтем правую часть равенства из левой; используя оценку $\sum_{i=1}^{k} \# H_{i} \geqslant 10 k$, получаем

$$
3 \sum_{i=1}^{k} \# H_{i}+(C-1) \# H-30 k \geqslant(C-1) \# H>0,
$$

что противоречит предыдущему равенству.

Предложение доказано. Доказательство теоремы 1.1 завершено.

\section{СПИСОК ЦИТИРОВАННОЙ ЛИТЕРАТУРЫ}

[1] Reid M. Young person's guide to canonical singularities // Algebraic geometry, Bowdoin, 1985 (Brunswick, Maine, 1985), Proc. Sympos. Pure Math. V. 46, Part 1. Providence, RI: Amer. Math. Soc., 1987. P. 345-414.

[2] Reid M. Canonical 3-folds // Journées de Géometrie Algébrique d'Angers, Juillet 1979. Algebraic Geometry, Angers: Sijthoff \& Noordhoff, 1979. P. 273-310.

[3] Mori S. On 3-dimensional terminal singularities // Nagoya Math. J. 1985. V. 98. P. 43-66.

[4] Morrison D., Stevens G. Terminal quotient singularities in dimensions three and four // Proc. Amer. Math. Soc. 1984. V. 90. № 1. P. 15-20.

[5] Mori S., Morrison D., Morrison I. On four-dimensional terminal quotient singularities // Math. Comp. 1988. V. 51. № 184. P. 769-786.

[6] Markushevich D., Prokhorov Yu. G. Exceptional quotient singularities // Amer. J. Math. 1999. V. 121. №6. P. 1179-1189.

[7] Спрингер Т. Теория инвариантов. М.: Мир, 1981. 